the glands or how it is formed, but the amount of dye absorbed from the letters written on the linen must be minute.

\section{THE RELATION OF ALCOHOLISM TO SUICIDE.}

Av interesting contribution on the above subject from the pen of Dr. W. C. Sullivan, of Her Majesty's Prison at Pentonville, has appeared in a recent number of the Journal of Mental Science (April, 1900). An attempt is made "to determine the rôle which alcoholism may have played in the late increase of suicide in England, and at establishing the distinctive characters which constitute the type of alcoholic suicide." In the classical work of Dr. Magnus Huss, that distinguished observer states " that the suicidal impulse is a more frequent accompaniment of the melancholia of drunkards than of melancholia from other causes; and further, that among the uneducated classes suicide frequently follows on the disordered emotional tone, which, sooner or later, results from the abuse of alcoholic liquors." The majority of observers have adopted the view which regards alcoholism as one among the more potent causes of suicide. Thus Lunier, whose views are in the main adopted by Morselli, found in the different departments of France that a high average consumption of alcohol was invariably accompanied by a high rate of suicide. On the other hand Baer, whose authority on the question is undoubted, has demonstrated the absence of such a correspondence in Prussia, and cites also as a counter-argument the case of Sweden, where decrease in alcoholism has failed to arrest the upward movement of suicide. Grotjahn, who regards alcoholism mainly as a direct result of the condition of the proletariat under the capitalistic régime, while admitting a certain coincidence in the regional and periodic distribution of alcoholism and suicide, regards their relationship as that of co-effects of a common cause. Dr. Sullivan, after a brief consideration of these and other writers, concludes that alcoholism is but one of the several causes of suicide, and that its relative importance as a suicidigenous factor differs in different places and at different epochs. Taking as the measure of alcoholism the deaths attributed to intemperance, which amounted to 35 per million in the Registrar-General's returns for 1867 , and which has steadily risen in successive periods till it stood at 76 per million in the returns for 1897 , it appears that an increase of alcoholism in the 30 years (1867-1897) amounts to over 100 per cent. "The records of coroners' verdicts of 'death from excessive drinking' shows a similar increase." A careful study also of the returns in the appendix to Mr. Whittaker's Memorandum, published with the report of the Licensing Commission, gives the average consumption per head of beer and spirits in the United Kingdom for the years 1842-98. From the figures given it appears that such consumption "has of late years shown an upward tendency, so marked as to bring the figures for recent years almost up to the level reached in the early seventies, when (coincident with the feverish industrialism of the period, and the enormous multiplication of licenses) English drinking habits attained their highest point." As regards the recent movement of suicide the Registrar-General's returns show that in England there has been a steady increase in the suicide rate during the last three decennial periods. Thus the proportion per million, which in the decennium 1861-70 stood at 65 , rose in the following decade to 70 ; and in the decade 1881-90 amounted to 77 , representing an increase of over 18 per cent. on the figures of the first-named period. As regards attempts to commit suicide, there has been a similar and even more decided increase. Thus in the period 1867-71 the number of cases of attempted suicide amounted to $35^{\circ} 5$ per million inhabitants; in each successive quinquennial period it stood -higher, and in the period $1892-96$ it rose to $57 \cdot 9$ per million-an increase of over 78 per cent. on the firstcited figures. Diagrams are given from the yearly returns, $1874-97$, to illustrate the increase of both actual suicides and suicidal attempts, and Dr. Sullivan shows "firstly, that suicidal tendencies have grown in a degree entirely out of proportion to the increase of population ; and secondly, that their growth has been much more considerable in the category of suicidal attempts than in that of actual suicides." As regards age and sex it is found that the proportion of suicides by persons aged over 45 years is 55.6 per cent., the period of maximum incidence being the decade 45.55 years of age. On the other hand the proportion of suicidal attempts among adult males fined by law for such showed that the period of maximum incidence was the decade $30-40$. A similar contrast is found as regards females; the maximum incidence in actual suicides being found in the decade $35-45$, while attempts to commit suicide prevail in the period 21-30. Hence it appears that abortive suicidal attempts tend to operate at a relatively early age. From Dr. Tatham's tables of occupational groups with a high rate of alcoholism, viz., publicans, butchers, coach and cab service, commercial travellers, hairdressers, and musicians, it appears that these groups also furnish a relatively high rate of suicide. From a careful discussion of the facts Dr. Sullivan draws the following conclusions :- Firstly, that the recent increase of suicide has coincided with a considerable development of abortive suicidal attempts. These attempts, in such of their characters as are ascertainable, have approximated to the type of alcoholic suicide, thereby confirming the clinical evidence which attributes to alcoholism the chief rôle in the genesis of abortive suicidal impulses. Second, that this increase of suicide has been in a large degree related to the influence of alcoholism, an influence which in the same period (as mortality statistics attest) has tended to augment. Third, that there is a type of alcoholic suicide,'with characteristics distinguishing it from suicide of other causation, and that, as compared with cases of deliberate and coordinated suicide due to other causes, alcoholic suicide is found to be more impulsive, more directly and immediately related to organic conditions in the individual. Fourth, the chronic intoxication by alcohol, as we observe it clinically, produces generalised disorders of visceral function throughout the economy, whence there results an alteration and disturbance of those organic stimuli which form the groundwork of our personality; those. stimuli whose activity, as Maudsley puts it, is "even of more consequence in determining the tone of our feeling 
or of our disposition and the character of our impulses than that activity which follows impressions received from the external world." The depressed emotional tone thereby induced prepares the suicidal impulse, which issues in action when a supervening increase of intoxication has still further lowered the level of function in the brain.

\section{THE RADICAL TREATMENT OF POLYPOID}

\section{DISEASE OF THE NOSE.}

NASAL polypi occur in a variety of forms, sometimes a single polyp of large or small dimensions arises without any associated suppurative discharge. Such cases are probably due to some undetermined interference with the vascular structures of the mucosa, with consequent odematous infiltration of the overlying submucous tissue. In course of time, from the accumulation of fluid, the polyp projects from the surface of the mucous membrane, and, perhaps partly owing to the force of gravity, the outgrowth assumes a pedunculated shape, and this, with the increasing size of the tumour, assumes the common form of a nasal polyp. Such growths may be removed by snare or otherwise, the base cauterised, and the patient permanently relieved.

But when with suppuration of the ethmoidal cells, and various pathological changes in the lining mucosa and the bony walls, numerous polypi are formed, the outlook is very different. The removal of the polypi give tempurary relief to the nasal obstruction, but the relief is transient only, for again and again, so long as the suppurative discharge persists, the polypi continue to recur. Moreover, the disease almost always tends to spread by continuity to adjacent nasal accessory cavities. The ultimate result is one of grave danger, not only to the health, but to the life of the patient, more especially when the frontal or the sphenoidal sinus become implicated. Hence it is a matter of very considerable moment that the common multiple nasal polypi with ethmoidal cell disease should receive greater care and more radical treatment than the simpler varieties already alluded to. For the severer and graver form it is not sufficient to remove the polypi and leave the underlying condition to run its course. Hence the method of radical treatment by curettement of the diseased areas becomes a very necessary procedure in many cases. It is often a difficult, and sometimes a dangerous operation, and requires an expert hand; but with due precaution and experience in the treatment of intranasal disease it is generally possible to obtain a lasting cure. Needless to say, it is necessary to recognise and to treat by corresponding radical methods suppuration emanating from the various accessory sinuses which may be concurrent. But we venture to think that hitherto the desirability of radical methods is not sufficiently appreciated by some practitioners, and while milder methods are not to be decried for many polypus cases, the possibility of newer methods offering a better chance of complete and permanent recovery should have due consideration.

\section{FEEDING IN GASTRIC ULCER.}

OFTEN enough the medical attendant finds much difficulty in arriving at a suitable dietary in the treatment of gastric ulcer; the indications are plainly to administer food only in such form as will not irritate the heating ulcer, but in practice this desirable end is not seldom a hard problem. Of course the difficulties may be obviated, for a time, by rectal feeding, but this process cannot be continued for any lengthy period without impairment to the general health of the patient. Sir Lauder Brunton's recent clinical lecture" on "Feeding in Gastric Ulcer" contains many invaluable practical hints which it is well to bear in mind. That such hard, indigestible or irritating substances as seeds, the achenia of strawberries, nuts, butcher meat, currants, pepper, etc., must be avoided is well recognised by medical practitioners, and reliance is placed chiefly on fluid foods. But it is not always sufficiently recognised that even such a simple fluid food as milk may result in the formation of hard indigestible coagula in the stomach. Brunton relates an instance where a female patient vomited a hard felt-like concretion, and another case where a similar fibrous concretion was removed per rectum, and in each case the concretion was formed of long curds of casein matted together, being the result of the rapid ingestion of milk. Hence the advantage of adding lime water to the milk, or of peptonising it so as to avoid those large indigestible curds being formed.

The next point of great practical moment which Brunton emphasises is the fact thai the longer food remains in the stomach the more acid does it become. We should therefore be careful to administer the food in small quantities at frequent intervals, rather than in three or four meals of larger quantity.

Further, the greater ease with which the digestion of pounded fish or meat as compared with the small solid masses brought about by mincing or mastication is a point to remember when a more extended diet is to be allowed.

Lastly, the proper way of preparing starchy food is touched upon, and we are reminded that we should beat the starch up with a little cold water, and then, when it is a paste, hot water may be added without causing lumpiness, while no one should be ignorant of the indigestibility of new bread as compared with stale.

Brit. Med. Journ. Mar. 1, 1902.

\section{PROGRESS IN OBSTETRICS.}

\section{(Continued from page 390.)}

Uterine Retraction.-Dr. Berry Hart, ${ }^{4}$ in a paper on this subject, deals with uterine retraction with special reference to the mechanism and management of the third stage of labour. He holds the view that the placenta is separated not during the "pain," but after it, and therefore advocates an expectant treatment during separation in the third stage of labour. By uterine retraction he understands the thickening which the uterine muscle retains after the contraction or "pain" is over. There is less retraction and 